\title{
Regeneration and adult stem cells in the human female reproductive tract
}

\author{
Masanori Ono \\ Tetsuo Maruyama \\ Yasunori Yoshimura \\ Department of Obstetrics and \\ Gynecology, Keio University School \\ of Medicine, Shinjuku, Tokyo, Japan
}

\begin{abstract}
The human uterus is unique in that it exhibits a tremendous regenerative capacity that enables cyclical regeneration and remodeling throughout a woman's reproductive life. This plasticity of the reproductive system has recently been highlighted. Regeneration and remodeling in the female reproductive tract alludes to the existence of endometrial and myometrial stem cell systems, which has been supported by increasing experimental evidence. Characterization of these stem cells, along with the study of the mechanisms controlling their regeneration, will improve the understanding of the physiology and pathophysiology of the female reproductive tract.
\end{abstract}

Keywords: adult stem cells, endometrium, myometrium, regeneration

\section{Introduction}

The derivation of human embryonic stem (hES) cells and induced pluripotent stem (iPS) cells has heralded a new era in stem cell research (Shamblott et al 1998; Thomson et al 1998; Takahashi et al 2007). Candidate stem cells have now been identified throughout the reproductive system and include the germ stem cells in the testis (Kanatsu-Shinohara et al 2004; Seandel et al 2007), as well as recently identified cell populations with adult stem cell activity in the human myometrium and endometrium (Gargett 2007; Ono et al 2007). The discovery of those cells highlights the importance of the stem cell system in human reproduction. The study of the adult stem cells derived from the female reproductive tract, in particular, is a new avenue through which to investigate gynecological diseases including leiomyoma, endometriosis, and endometrial cancer.

The human uterus is unique in that it possesses the tremendous regenerative capacity required for cyclical regeneration and remodeling throughout reproductive life. Not only must the uterus rapidly enlarge to accommodate the developing fetus, the endometrium must also regenerate with each menstrual cycle. Regeneration and remodeling in the female reproductive tract allude to the existence of myometrial and endometrial stem cell systems; however, the characteristics and function of these stem cells remain poorly understood.

The concept that endometrial regeneration is mediated by endometrial stem/ progenitor cells was proposed many years ago (Prianishnikov 1978; Padykula et al 1989). Since then, indirect evidence has accumulated from proliferation studies, clinical observations, and the demonstration of gland monoclonality (Gargett 2007). Shedding of the endometrial functionalis layer at menstruation and its subsequent regeneration from the endometrial basalis suggests that the proliferation kinetics differ between the two layers (Brenner et al 2003) and that putative endometrial stem cells reside in the basalis. Given that endometriotic lesions have a basalis phenotype (Leyendecker et al 2002), it is likely that adult stem cells present in the basalis may implant ectopically through retrograde menstruation and may give rise to 
endometriotic lesions (Sasson and Taylor 2008). Since the endometrium is comprised of glands, surface epithelium and supportive stroma, it is plausible that there may exist both epithelial and stromal stem/progenitor cells responsible for the regenerative capacity of the endometrium (Chan et al 2004; Gargett 2007).

Our recent xenograft study confirms the plasticity of endometrial cells. We have shown that a small number of singly dispersed human endometrial cells, transplanted beneath the kidney capsule of severely immunodeficient mice, regenerates functional endometrial tissue (Masuda et al 2007). This artificially generated endometrium resembles the natural endometrium and contains human blood vessels which invade the mouse kidney parenchyma. Additionally, it mimics normal hormone dependent changes including proliferation, differentiation, and tissue breakdown (menstruation).

We have also recently isolated a candidate population of adult stem cells from the human myometrium. Selection was performed using a side population method based on a distinct Hoechst dye efflux pattern (Ono et al 2007). Characterization of these myometrial cells, along with the study of the mechanisms controlling their regeneration, will improve the understanding of the physiology and pathophysiology of the female reproductive tract.

\section{Human endometrial stem/ progenitor cells}

Stem cells are rare undifferentiated cells present in many adult tissues. Their rarity and lack of distinguishing morphological features and specific markers make it difficult to identify their location in tissues. Rather, adult stem cells are defined by their functional properties: substantial self-renewal capacity, and ability to differentiate into at least one type of mature functional progeny (Figure 1).

Investigators have attempted to identify endometrial stem cells with conventional methods employing known stem cell markers, as well as with several functional studies designed to identify populations of cells with stem cell activity. The latter approach has proved more fruitful.

The first published evidence for the existence of endometrial stem/progenitor cells comes from a recent study that identified clonogenic human endometrial epithelial and stromal cells (Chan et al 2004). Clonogenicity, the ability of a single cell to initiate a colony of cells when cultured at extremely low seeding densities, is a classic

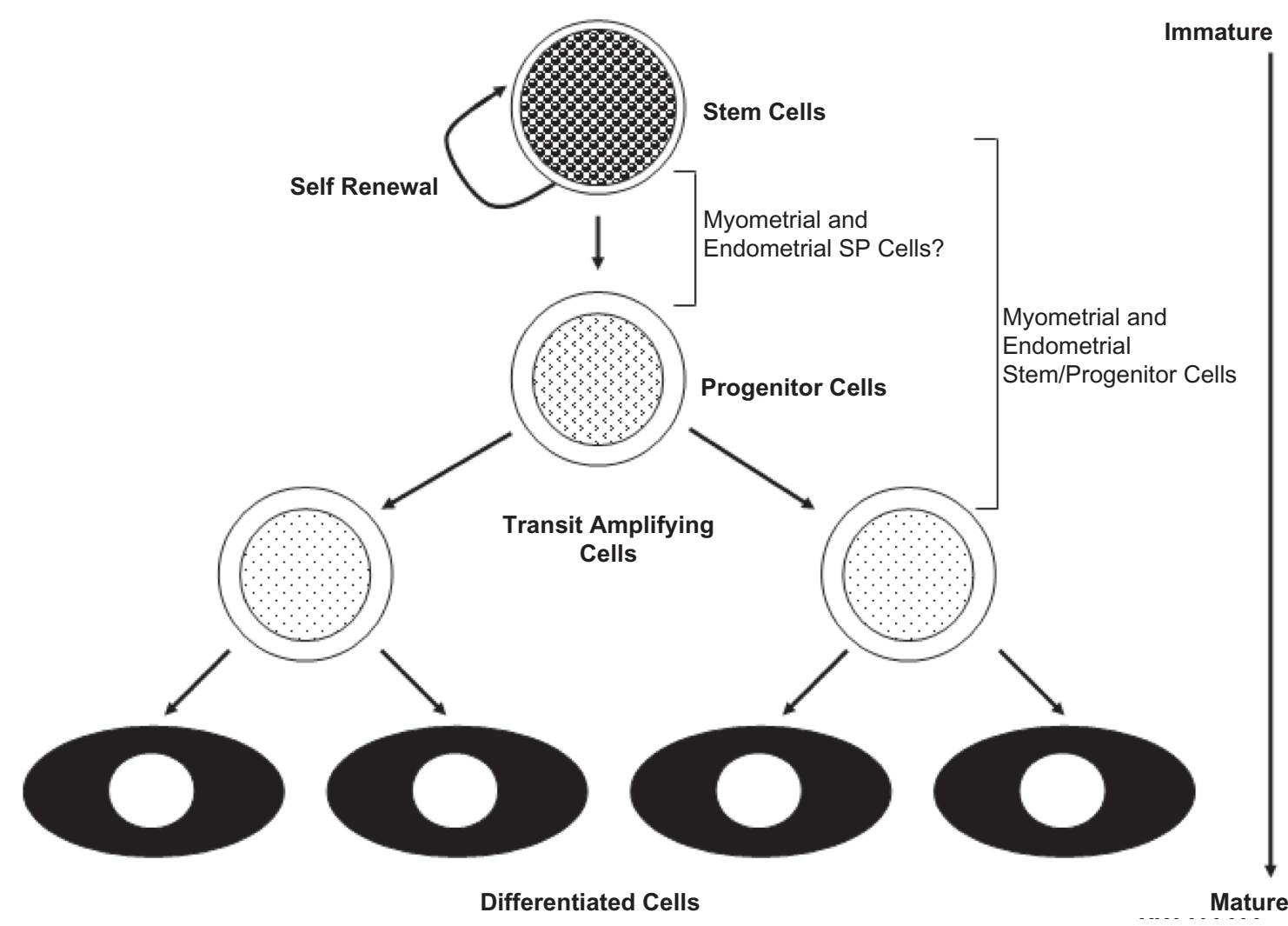

Figure I The relationship of putative endometrial and myometrial stem/progenitor cells to the hierarchical model of stem cell differentiation. 
in-vitro stem/progenitor cell property. Clonogenicity has been demonstrated for many adult stem cell types (van Os et al 2004; Gargett 2007). Human endometrial stromal cells are significantly more clonogenic than endometrial epithelial cells, although both cell types form large and small colonies (Chan et al 2004). Substantial variability in clonogenicity is observed between samples for both epithelial and stromal cells (Chan et al 2004; Schwab et al 2005). This may be a reflection of cellular changes related to menstrual cycle stages. Endometrial stromal cells demonstrate a trend for higher clonogenicity in the proliferative stage, whereas for epithelial cells, this occurs in the secretory stage (Schwab et al 2005). Self-renewal or the ability to produce identical daughter stem cells is required to maintain the stem cell pool in tissues. Asymmetric cell division is one mechanism for producing an identical daughter cell and more differentiated daughter. However, stem cells also undergo symmetric divisions, either producing daughter stem cells or transit amplifying progenitors. In summary, these studies identifying clonogenic epithelial and stromal cells suggest that two types of stem/progenitor cell exist in the human endometrium. They are present in different proportions, have differing growth factor requirements, and are likely to have specific niches in the endometrium (Chan et al 2004; Schwab et al 2005; Gargett 2006, 2007) Current studies examining stem cell attributes of the rare epithelial and stromal cells have demonstrated their high proliferative potential as they undergo 30-32 population doublings before senescence or transformation (Gargett 2007). Human endometrial clonogenic cells exhibit multilineage differentiation and similar to bone marrow and adipose tissue MSC differentiating into mesenchy, al lineages; adipocytes, smooth muscle cells, chondrocytes and osteoblasts, in vitro. This suggests that the rare endometrial clonogenic cells have characteristic properties of endometrial stem cells which are probably responsible for the remarkable, cyclical and regenerative capacity of human endometrium (Gargett 2006) Additionally, cells derived from myometrium have differentiation capacity not only into myocytes but also into adipocytes and osteocytes (Ono et al 2007).

Side population (SP) cells have recently been identified in short-term cultures of cell fractions, filtered from human endometrial cell suspensions (Kato et al 2007). SP cells are a small fraction of cells within tissues with the unique ability to efflux the DNA-binding dye Hoechst 33342 via the ATP-binding cassette transporter G2 (ABCG2) (Goodell et al 1996; Challen and Little 2006). They are detected as poorly stained cells by dual wavelength flow cytometric analysis after incubation with Hoechst 33342 . SP cells have been isolated from various adult tissues, demonstrating that this phenotype may represent a common feature of adult stem cells. (Montanaro et al 2003; Matsuzaki et al 2004; Redvers et al 2006; Schienda et al 2006) The percentage of side population cells is highly variable between samples, although it is significantly greater during menstruation than in the proliferative and secretory stages (Kato et al 2007). This is perhaps due to loss of the functionalis. Though the percentages of side population cells and clonogenic cells are similar, it is not known whether the SP fraction is clonogenic (Chan et al 2004; Schwab et al 2005; Kato et al 2007).

Three studies have examined the expression of stem cell markers in the human endometrium (Cho et al 2004; Matthai et al 2006; Kato et al 2007). These studies, while valuable, require further analyses to validate whether cells expressing these markers function as endometrial stem/progenitor cells. Oct-4, a transcription factor and marker of human embryonic stem cells, and more recently of adult stem cells, is expressed in almost half of tested endometrial samples (Cho et al 2004; Tai et al 2005). More Oct-4+ cells are observed during the proliferative stage; however, the identity and location of the Oct-4+ cells have not been reported (Matthai et al 2006). Several general adult stem cell markers including bcl-2, c-kit (CD117) and CD34, have also been identified in endometrial tissues (Cho et al 2004). The importance of these markers, however, cannot be determined since they are expressed in many more endometrial cells than the numbers of clonogenic or side population cells identified in functional studies (Chan et al 2004; Kato et al 2007). Thus the identification of markers specific to the endometrial stem/progenitor cell population is an area for future investigation.

Xenografts of human endometrial tissue fragments into immunocompromised mice have been used as a model to investigate the pathophysiology of endometriosis (Grummer 2006). More recently, two studies have demonstrated growth of human endometrial tissue from dissociated endometrial cells transplanted into immunocompromised mice (Kurita et al 2005; Masuda et al 2007). Endometrial tissue may be reconstructed from tissue recombinants of mouse neonatal mesenchyme or human endometrial stromal cells and human gland micro-organoids transplanted into nude mice lacking T lymphocyte activity (Kurita et al 2005). Mixed populations of human endometrial epithelial and stromal fractions, transplanted beneath the kidney capsule, a richly vascularized tissue, demonstrate the importance of epithelial-stromal interactions in generating endometrial glandular tissues. Fully dissociated human epithelial and stromal cells injected 
directly under the kidney capsule of NOD/SCID/ $\gamma \mathrm{c}$ null (NOG) immunodeficient mice, lacking T, B, and natural killer cell activity, produce well organized tissue recapitulating endometrial and myometrial layers (Masuda et al 2007). The glandular epithelial structures express progesterone receptors and proliferate in response to estrogen, while stromal cells differentiate into prolactin-producing decidual cells on further administration of progesterone (Masuda et al 2007). These models, particularly the NOG mice, are an excellent in-vivo assay system to test whether candidate human or mouse endometrial stem/progenitor cell populations reconstitute endometrial tissue.

Another retrospective approach to studying adult stem/ progenitor cell activity in the endometrium involves the analysis of methylation patterns in endometrial glands (Kim et al 2005). Epigenetic changes to DNA sequences, arising during cell division, encode a cellular history in individual glands. This history reflects the methylation patterns arising in resident stem/progenitor cells, since the changes are inherited in subsequent cell divisions and retained. In contrast, changes arising in more mature progeny are lost when these cells are shed. Mathematical models describing the methylation patterns observed in individual glands from cycling and atrophic human endometrium support the concept that an individual gland contains a stem cell niche. Within each niche is an unknown number of long-lived stem cells rather than a single stem cell (Kim et al 2005). Finally, gland diversity is maintained in the aging endometrium, which indicates that a reservoir of stem cells remains even when the endometrium is atrophic. This observation supports the data from the clonogenicity studies (Schwab et al 2005).

While it is possible that human endometrial epithelial and stromal stem/progenitor cells may be derived from residual fetal stem cells (Gargett 2007), one study suggests that the bone marrow is a potential source (Taylor 2004). Significant chimerism, ranging from $0.2 \%$ to $52 \%$, is detectable in the endometrial glands and stroma of women who have received single-antigen, HLA-mismatched, bone marrow transplants. This suggests that bone marrow stem cells contribute to endometrial regeneration in the setting of cellular turnover and inflammatory stimuli (Taylor 2004). Most gland profiles are exclusively of the donor or host type, although there is some chimerism within individual glands. This suggests that not all are monoclonal, consistent with the methylation pattern data of individual glands (Kim et al 2005). In contrast, the endometrial glands found in nontransplanted women are all monoclonal (Tanaka et al 2003). These studies do not include information on the type of bone marrow transplant or the reproductive history; thus it is not possible to determine the source of the chimeric endometrial cells. In the case of women who received transplants as children, stem cells from their mothers may persist, as bidirectional cell trafficking occurs during pregnancy. Thus the ultimate source of endometrial stem/progenitor cells is currently uncertain. Neither is it known whether bone marrow-derived or fetal-derived cells regularly engraft the endometrium under normal physiological conditions or if they incorporate into endometrial tissue in the basalis, functionalis, or both regions.

\section{Mouse endometrial stem cells}

Although specific markers of mouse endometrial epithelial and stromal/mesenchymal stem cells have not yet been identified, three recent studies have utilized the label-retaining cell (LRC) technique as a functional approach for identifying candidate adult stem cells in vivo (Chan and Gargett 2006; Cervello et al 2007; Szotek et al 2007). This technique identifies stem cells by their quiescent, slowly cycling nature. In the three LRC studies, mouse uteri were pulse-labeled with bromodeoxyuridine (BrdU), a DNA synthesis label, during a time in which all cells, including stem cells, were dividing. This ensured that the majority of cells were labeled. Labeling was followed by a chase period in which the label was diluted out through numerous cell divisions in the proliferating cells, but was retained in a small population of infrequently cycling stem/progenitor cells, or label retaining cells (Braun and Watt 2004). Immunohistochemistry was used to localize BrdU+ epithelial, stromal, and myometrial LRC, revealing their locations and proportions in the stem cell niche. All three studies identified a stromal LRC population (Chan and Gargett 2006; Cervello et al 2007; Szotek et al 2007).

The LRCs have been further characterized based on the expression of various markers. Both epithelial and stromal LRCs are CD45- (Chan and Gargett 2006; Szotek et al 2007). This indicates they are not leukocytes and suggests that under normal physiological conditions, the source of mouse endometrial stromal stem/progenitor cells is not the bone marrow. A small population of stromal LRCs expresses the key stem cell markers, c-kit and Oct-4 (Cervello et al 2007). These cells are predominantly located near the endometrial myometrial interface, suggesting that this subpopulation may contain stromal stem/progenitor cells.

\section{Human myometrial stem cells}

Over the course of pregnancy, the human uterus undergoes a 500 - to 1,000-fold increase in volume and a 24 -fold increase 
in weight. The uterine smooth muscle layer, or myometrium, is remodeled through a combination of cell hypertrophy and hyperplasia. The origin of the new smooth muscle cells, however, is unclear. They may arise from existing smooth muscle cells, or they may be the product of stem cell differentiation. The human uterus, which is composed mainly of myometrial cells, exhibits an expansion in size over the course of pregnancy. Both myometrial hyperplasia (an increase in cell number) and hypertrophy (an increase in cell size) contribute to the dramatic growth of the pregnant uterus (Ramsey 1994; Shynlova et al 2006). In humans, most growth results from stretch-induced myometrial hypertrophy. Uterine growth during the first weeks of pregnancy, however, is accomplished by myometrial hyperplasia with a smaller contribution from hypertrophy (Ramsey 1994). Similarly in rats, myometrial hyperplasia is high during early gestation and decreases dramatically later; and myometrial hypertrophy is low at the beginning of pregnancy but increases as gestation progresses (Shynlova et al 2006). These changes repeat with each successive pregnancy. The presence of stem cells in other areas of the body that undergo continual renewal such as the bone marrow, gut, and skeletal muscle suggests that the changes in the uterus may not be attributable to the hypertrophy and hyperplasia of existing myometrial cells alone (Körbling and Estrov 2003).

The human subendometrial myometrium originates from the Müllerian duct, whereas the outer myometrium has a non-Müllerian origin (Gargett 2007); however, both are derived from the mesenchyme (Konishi et al 1984). Mesenchymal stem cells (MSCs) are defined as selfrenewable, multipotent progenitor cells with the capacity to differentiate into several distinct mesenchymal lineages (He et al 2007). Recently, a SP population of cells has also been identified in the myometrium. However, there exist some phenotypic and functional differences between the myometrial SP (myoSP) and MSCs. First, although myoSP express several MSC markers including CD90, CD73, CD105, and STRO-1, the majority of myoSP are CD34-positive and CD44-negative, whereas MSCs are negative for CD34 and positive for CD44 (Deans and Moseley 2000). Second, unlike bone marrow- or peripheral blood-derived MSCs (Wakitani et al 1995; Gang et al 2004), myoSP do not generate skeletal muscle cells when transplanted into intact or chemically injured skeletal muscles of NOG mice (Ono et al 2007). Further studies are required to elucidate why myoSP behaves differently from MSCs in response to the microenvironment and/or its niche in various tissues and organs. Interestingly, although myoSP never proliferates efficiently in vitro in a normoxic $\left(20 \% \mathrm{O}_{2}\right)$ environment, it proliferates efficiently in vitro under $2 \%$ oxygen tension.

Leiomyomas are the most common gynecological tumors in women of reproductive age, and are associated with a variety of symptoms including abnormal uterine bleeding, pelvic pain, urinary frequency, impaired fertility, and spontaneous abortion. They are clonal in origin (Walker and Stewart 2005) and their development is thought to be induced and promoted by hypoxia (Fukuhara et al 2002; Pavlovich and Schmidt $2004)$. Low oxygen tension $\left(1 \%-5 \% \mathrm{O}_{2}\right)$ dramatically upregulates secreted frizzled-related protein 1, a modulator of Wnt signaling. Secreted frizzled-related protein 1 exerts antiapoptotic effects in leiomyoma cells but not myometrial cells (Fukuhara et al 2002). Myometrial contraction and vasoconstriction that occur during menstruation render the myometrium hypoxic. It is possible that repeated menstruation-induced hypoxia may select a single cell such as a myoSP to proliferate and acquire cytogenetic abnormalities that would ultimately result in the development of a leiomyoma. Leiomyomas occasionally contain adipogenic components and are referred to as lipoleiomyomas (Wang et al 2006). Very rarely, they also become ossified, which is consistent with the potential of myoSP to differentiate not only into myocytes but also into adipocytes and osteocytes.

\section{Mouse myometrial stem cells}

Arango and colleagues (2005) reported that Müllerian duct mesenchyme-specific disruption of $\beta$-catenin resulted in a progressive turnover of the uterine myometrium to adipose tissue. This supports the possibility that putative myometrial stem cells, with the potential for differentiation into adipocytes in the absence of $\beta$-catenin, may exist in the myometrium. These cells may give rise to lipoleiomyomas. Indeed, the same group has recently isolated a myometrial SP from the mouse uterus and provided evidence that this SP contains putative myometrial stem/progenitor cells derived from the Müllerian duct mesenchyme (Szotek et al 2007). This finding supports our results on the enrichment of stem cells in myoSP in humans (Ono et al 2007). The embryonic Müllerian ducts, or paramesonephric ducts, are derived from the coelomic epithelium in the bilateral urogenital ridges, during differentiation of the bipotential gonad (Teixeira et al 2001). In female embryos, the absence of Müllerian inhibiting substance (MIS) allows the Müllerian ducts to persist and to differentiate into the internal female reproductive tract structures (Teixeira et al 2001). After birth, the mesenchyme of the primitive uterine tube differentiates into two layers of 
the adult uterus: the endometrial stroma and the myometrial muscle layers (Kurita et al 2001). By postnatal day 15, the myometrium is well developed and the endometrial glands are visibly coiled, comparable to those observed in the adult uterus.

The adult uterus undergoes repeated cycles of cellular proliferation and degeneration in response to hormonal signals during a normal mammalian reproductive life span. These changes involve signaling through $\beta$-catenin. $\beta$-catenin has two roles: as an intracellular transcriptional cofactor of the canonical Wnt signaling cascade and as a structural adaptor protein linking cadherins to the actin cytoskeleton in cell-cell adhesion (Nelson and Nusse 2004). The progressive smooth muscle atrophy and resultant adipogenesis in the conditional $\beta$-catenin-deleted myometrium suggests that this cell fate switch is likely due to the dysregulation of a previously uncharacterized, intrinsic, myometrial maintenance and repair mechanism. This mechanism presumably involves perturbed stem or progenitor cell activity.

Cell lineage and early differentiation markers in the mouse myometrium are largely uncharacterized. Although much effort has focused on the events surrounding the endometrium during the estrous cycle and at pregnancy, little is known about the normal mechanisms of repair and remodeling in the myometrium. Stromal LRCs are present near the endometrial epithelium, in proximity to the myometrial-stromal junction, and in a perivascular distribution (Chan and Gargett 2006). Myometrial LRCs are present at the periphery of the longitudinal muscle bundles, a location similar to where Pax7-expressing satellite or stem cells in skeletal muscle are found. In vitro and in vivo evidence suggests that either of the two known biological functions of $\beta$-catenin, Wnt signaling and cell-cell junctions, may be involved in the muscle-to-fat cell fate switch (Ross et al 2000; Kennell and MacDougald 2005) Disruption of the Wnt canonical signaling pathway induces adipogenesis and causes the transdifferentiation of myoblasts into adipocytes. The mechanism of this likely involves the disruption of $\beta$-catenin-mediated inhibition of adipogenic transcription factors (Ross et al 2000). Another possibility is that $\beta$-catenin cell-cell junctions play a role in the maintenance of the myometrial stem cell niche (Szotek et al 2007). Disruption of this niche may result in a cell fate switch from smooth muscle myogenesis to adipogenesis.

\section{Perspective}

There is now sufficient published evidence to conclude that rare populations of adult stem cells exist in the human myometrium and endometrium. The study of stem cells in the female reproductive tract, however, is still in its infancy. There is a pressing need to identify definitive markers for both myometrial and endometrial stem cells. A thorough characterization of uterine stem cells is a prerequisite for understanding the complex mechanisms underlying the morphogenesis and physiological regeneration of the female reproductive tract. Additionally, the techniques developed by our laboratory for culturing and differentiating uterine stem cells could be a starting point for using these cells for the regeneration of the uterus or other organs. Studying the underlying mechanisms involved in the uterine stem cell niche will improve the understanding of the pathophysiology of uterine cancer, hyperplasia, endometriosis, leiomyomas, and adenomyosis, and may change how these diseases are treated in the future.

\section{Disclosure}

The authors report no conflicts of interest in this work.

\section{References}

Arango NA, Szotek PP, Manganaro TF, et al. 2005. Conditional deletion of beta-catenin in the mesenchyme of the developing mouse uterus results in a switch to adipogenesis in the myometrium. Dev Biol, 288:276-83.

Braun KM, Watt FM. 2004. Epidermal label-retaining cells: background and recent applications. J Investig Dermatol Symp Proc, 9:196-201.

Brenner RM, Slayden OD, Rodgers WH, et al. 2003. Immunocytochemical assessment of mitotic activity with an antibody to phosphorylated histone $\mathrm{H} 3$ in the macaque and human endometrium. Hum Reprod, 18:1185-93.

Cervello I, Martinez-Conejero JA, Horcajadas JA, et al. 2007. Identification, characterization and co-localization of label-retaining cell population in mouse endometrium with typical undifferentiated markers. Hum Reprod, 22:45-51.

Challen GA, Little MH. 2006. A side order of stem cells: the SP phenotype. Stem Cells, 24:3-12.

Chan RW, Gargett CE. 2006. Identification of label-retaining cells in mouse endometrium. Stem Cells, 24:1529-38.

Chan RW, Schwab KE, Gargett CE. 2004. Clonogenicity of human endometrial epithelial and stromal cells. Biol Reprod, 70:1738-50.

Cho NH, Park YK, Kim YT, et al. 2004. Lifetime expression of stem cell markers in the uterine endometrium. Fertil Steril, 81:403-7.

Deans RJ, Moseley AB. 2000. Mesenchymal stem cells: biology and potential clinical uses. Exp Hematol, 28:875-84.

Fukuhara K, Kariya M, Kita M, et al. 2002. Secreted frizzled related protein 1 is overexpressed in uterine leiomyomas, associated with a high estrogenic environment and unrelated to proliferative activity. $J$ Clin Endocrinol Metab, 87:1729-36.

Gang EJ, Jeong JA, Hong SH, et al. 2004. Skeletal myogenic differentiation of mesenchymal stem cells isolated from human umbilical cord blood. Stem Cells, 22:617-24.

Gargett CE. 2006. Identification and characterisation of human endometrial stem/progenitor cells. Aust N Z J Obstet Gynaecol, 46:250-3.

Gargett CE. 2007. Uterine stem cells: what is the evidence? Hum Reprod Update, 13:87-101.

Goodell MA, Brose K, Paradis G, et al. 1996. Isolation and functional properties of murine hematopoietic stem cells that are replicating in vivo. J Exp Med, 183:1797-806. 
Grummer R. 2006. Animal models in endometriosis research. Hum Reprod Update, 12:641-9.

He Q, Wan C, Li G. 2007. Concise review: multipotent mesenchymal stromal cells in blood. Stem Cells, 25:69-77.

Kanatsu-Shinohara M, Inoue K, Lee J, et al. 2004. Generation of pluripotent stem cells from neonatal mouse testis. Cell, 119:1001-12.

Kato K, Yoshimoto M, Kato K, et al. 2007. Characterization of side-population cells in human normal endometrium. Hum Reprod, 22:1214-23.

Kennell JA, MacDougald OA. 2005. Wnt signaling inhibits adipogenesis through beta-catenin-dependent and -independent mechanisms. J Biol Chem, 280:24004-10.

Kim JY, Tavare S, Shibata D. 2005. Counting human somatic cell replications: methylation mirrors endometrial stem cell divisions. Proc Natl Acad Sci U S A, 102:17739-44.

Konishi I, Fujii S, Okamura H, et al. 1984. Development of smooth muscle in the human fetal uterus: an ultrastructural study. J Anat, 139(Pt 2):239-52.

Körbling M, Estrov Z. 2003. Adult stem cells for tissue repair - a new therapeutic concept? N Engl J Med, 349:570-82.

Kurita T, Cooke PS, Cunha GR. 2001. Epithelial-stromal tissue interaction in paramesonephric (Mullerian) epithelial differentiation. Dev Biol, 240:194-211.

Kurita T, Medina R, Schabel AB, et al. 2005. The activation function-1 domain of estrogen receptor alpha in uterine stromal cells is required for mouse but not human uterine epithelial response to estrogen. Differentiation, 73:313-22.

Leyendecker G, Herbertz M, Kunz G, et al. 2002. Endometriosis results from the dislocation of basal endometrium. Hum Reprod, 17:2725-36.

Masuda H, Maruyama T, Hiratsu E, et al. 2007. Noninvasive and realtime assessment of reconstructed functional human endometrium in NOD/SCID/gamma c(null) immunodeficient mice. Proc Natl Acad Sci U S A, 104:1925-30.

Matsuzaki Y, Kinjo K, Mulligan RC, et al. 2004. Unexpectedly efficient homing capacity of purified murine hematopoietic stem cells. Immunity, 20:87-93.

Matthai C, Horvat R, Noe M, et al. 2006. Oct-4 expression in human endometrium. Mol Hum Reprod, 12:7-10.

Montanaro F, Liadaki K, Volinski J, et al. 2003. Skeletal muscle engraftment potential of adult mouse skin side population cells. Proc Natl Acad Sci US A, 100:9336-41.

Nelson WJ, Nusse R. 2004. Convergence of Wnt, beta-catenin, and cadherin pathways. Science, 303:1483-7.

Ono M, Maruyama T, Masuda H, et al. 2007. Side population in human uterine myometrium displays phenotypic and functional characteristics of myometrial stem cells. Proc Natl Acad Sci U S A, 104:18700-5.

Padykula HA, Coles LG, Okulicz WC, et al. 1989. The basalis of the primate endometrium: a bifunctional germinal compartment. Biol Reprod, 40:681-90.

Pavlovich CP, Schmidt LS. 2004. Searching for the hereditary causes of renal-cell carcinoma. Nat Rev Cancer, 4:381-93.

Prianishnikov VA. 1978. On the concept of stem cell and a model of functional-morphological structure of the endometrium. Contraception, $18: 213-23$.
Ramsey EM. 1994. Anatomy of the human uterus. Cambridge: Cambridge University Press.

Redvers RP, Li A, Kaur P. 2006. Side population in adult murine epidermis exhibits phenotypic and functional characteristics of keratinocyte stem cells. Proc Natl Acad Sci U S A, 103:13168-73.

Ross SE, Hemati N, Longo KA, et al. 2000. Inhibition of adipogenesis by Wnt signaling. Science, 289:950-3.

Sasson IE, Taylor HS. 2008. Stem cells and the pathogenesis of endometriosis. Ann N Y Acad Sci, 1127:106-15.

Schienda J, Engleka KA, Jun S, et al. 2006. Somitic origin of limb muscle satellite and side population cells. Proc Natl Acad Sci U S A, 103:945-50.

Schwab KE, Chan RW, Gargett CE. 2005. Putative stem cell activity of human endometrial epithelial and stromal cells during the menstrual cycle. Fertil Steril, 84(Suppl 2):1124-30.

Seandel M, James D, Shmelkov SV, et al. 2007. Generation of functional multipotent adult stem cells from GPR125+ germline progenitors. Nature, 449:346-50.

Shamblott MJ, Axelman J, Wang S, et al. 1998. Derivation of pluripotent stem cells from cultured human primordial germ cells. Proc Natl Acad Sci U S A, 95:13726-31.

Shynlova O, Oldenhof A, Dorogin A, et al. 2006. Myometrial apoptosis: activation of the caspase cascade in the pregnant rat myometrium at midgestation. Biol Reprod, 74:839-49.

Szotek PP, Chang HL, Zhang L, et al. 2007. Adult mouse myometrial label-retaining cells divide in response to gonadotropin stimulation. Stem Cells, 25:1317-25.

Tai MH, Chang CC, Kiupel M, et al. 2005. Oct4 expression in adult human stem cells: evidence in support of the stem cell theory of carcinogenesis. Carcinogenesis, 26:495-502.

Takahashi K, Tanabe K, Ohnuki M, et al. 2007. Induction of pluripotent stem cells from adult human fibroblasts by defined factors. Cell, 131:861-72.

Tanaka M, Kyo S, Kanaya T, et al. 2003. Evidence of the monoclonal composition of human endometrial epithelial glands and mosaic pattern of clonal distribution in luminal epithelium. Am J Pathol, 163:295-301.

Taylor HS. 2004. Endometrial cells derived from donor stem cells in bone marrow transplant recipients. JAMA, 292:81-5.

Teixeira J, Maheswaran S, Donahoe PK. 2001. Mullerian inhibiting substance: an instructive developmental hormone with diagnostic and possible therapeutic applications. Endocr Rev, 22:657-74.

Thomson JA, Itskovitz-Eldor J, Shapiro SS, et al. 1998. Embryonic stem cell lines derived from human blastocysts. Science, 282:1145-7.

van Os R, Kamminga LM, de Haan G. 2004. Stem cell assays: something old, something new, something borrowed. Stem Cells, 22:1181-90.

Wakitani S, Saito T, Caplan AI. 1995. Myogenic cells derived from rat bone marrow mesenchymal stem cells exposed to 5-azacytidine. Muscle Nerve, 18:1417-26.

Walker CL, Stewart EA. 2005. Uterine fibroids: the elephant in the room. Science, 308:1589-92.

Wang X, Kumar D, Seidman JD. 2006. Uterine lipoleiomyomas: a clinicopathologic study of 50 cases. Int J Gynecol Pathol, 25:239-42. 
